University of Wollongong

Research Online

Faculty of Engineering - Papers (Archive)

Faculty of Engineering and Information

Sciences

2007

\title{
Growth of Ca3Co409 films: Simple chemical solution deposition and stress induced spontaneous dewetting
}

\author{
Xuebin Zhu \\ University of Wollongong, xuebin@uow.edu.au \\ Hechang Lei \\ Chinese Academy of Sciences \\ Yuping Sun \\ Chinese Academy of Sciences \\ Wenhai Song \\ Chinese Academy of Sciences \\ Bangchuan Zhao \\ Chinese Academy of Sciences
}

See next page for additional authors

Follow this and additional works at: https://ro.uow.edu.au/engpapers

Part of the Engineering Commons

https://ro.uow.edu.au/engpapers/2610

\section{Recommended Citation}

Zhu, Xuebin; Lei, Hechang; Sun, Yuping; Song, Wenhai; Zhao, Bangchuan; Li, Xianghu; Ang, Ran; Shi, Dongqi; and Dou, S X.: Growth of Ca3Co409 films: Simple chemical solution deposition and stress induced spontaneous dewetting 2007, 103519-1-103519-7.

https://ro.uow.edu.au/engpapers/2610 


\section{Authors}

Xuebin Zhu, Hechang Lei, Yuping Sun, Wenhai Song, Bangchuan Zhao, Xianghu Li, Ran Ang, Dongqi Shi, and SX. Dou 


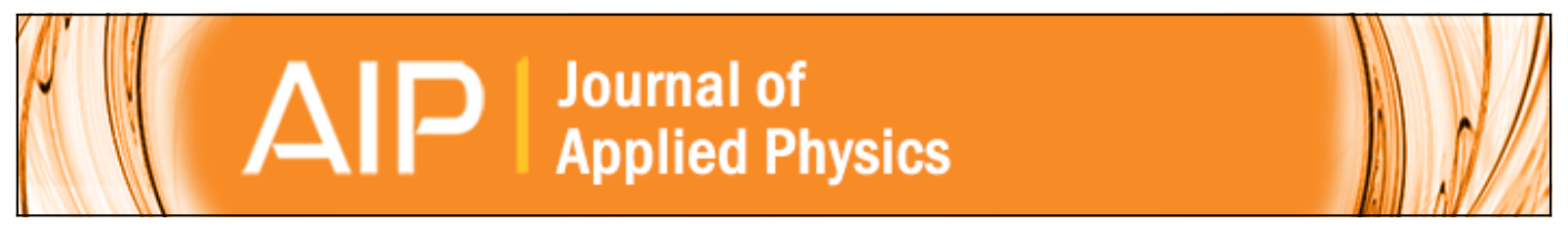

\section{Growth of Ca 3 Co 409 films: Simple chemical solution deposition and stress induced spontaneous dewetting}

Xuebin Zhu, Yuping Sun, Hechang Lei, Xianghu Li, Ran Ang, Bangchuan Zhao, Wenhai Song, Dongqi Shi, and Shixue Dou

Citation: Journal of Applied Physics 102, 103519 (2007); doi: 10.1063/1.2818109

View online: http://dx.doi.org/10.1063/1.2818109

View Table of Contents: http://scitation.aip.org/content/aip/journal/jap/102/10?ver=pdfcov

Published by the AIP Publishing

\section{Articles you may be interested in}

Enhanced high temperature thermoelectric properties of Bi-doped c -axis oriented Ca 3 Co $4 \mathrm{O} 9$ thin films by pulsed laser deposition

J. Appl. Phys. 108, 083709 (2010); 10.1063/1.3499324

Metallic conductivity at the $\mathrm{Ca} \mathrm{Hf} \mathrm{O} 3 \mathrm{Sr} \mathrm{Ti} O 3$ interface

Appl. Phys. Lett. 91, 232106 (2007); 10.1063/1.2816907

Thermoelectric properties and microstructure of c -axis-oriented Ca 3 Co 4 O 9 thin films on glass substrates Appl. Phys. Lett. 87, 171912 (2005); 10.1063/1.2117615

In situ growth of c -axis-oriented Ca 3 Co 4 O 9 thin films on Si (100)

Appl. Phys. Lett. 86, 082103 (2005); 10.1063/1.1868873

Influence of pulsed laser deposition growth conditions on the thermoelectric properties of Ca 3 Co 4 O 9 thin films

J. Appl. Phys. 97, 013706 (2005); 10.1063/1.1823582

\section{A|P| Journal of}

Journal of Applied Physics is pleased to announce André Anders as its new Editor-in-Chief 


\title{
Growth of $\mathrm{Ca}_{3} \mathrm{Co}_{4} \mathrm{O}_{9}$ films: Simple chemical solution deposition and stress induced spontaneous dewetting
}

\author{
Xuebin Zhu ${ }^{\text {a) }}$ \\ Key Laboratory of Materials Physics, Institute of Solid State Physics, Chinese Academy of Sciences, \\ Hefei 230031, People's Republic of China \\ and Institute for Superconducting and Electronic Materials, University of Wollongong, \\ New South Wales 2522, Australia \\ Yuping Sun, Hechang Lei, Xianghu Li, Ran Ang, Bangchuan Zhao, and Wenhai Song \\ Key Laboratory of Materials Physics, Institute of Solid State Physics, Chinese Academy of Sciences, \\ Hefei 230031, People's Republic of China \\ Dongqi Shi and Shixue Dou \\ Institute for Superconducting and Electronic Materials, University of Wollongong, New South Wales 2522, \\ Australia
}

(Received 15 April 2007; accepted 5 October 2007; published online 30 November 2007)

\begin{abstract}
Chemical solution deposition as a simple method for preparation of oxide films is used to fabricate misfit structured $\mathrm{Ca}_{3} \mathrm{Co}_{4} \mathrm{O}_{9}$ thermoelectric films using cheap precursors, $\mathrm{Ca}$ and $\mathrm{Co}$ acetates, which opens up a simple route to fabricate large-area Co-based oxide thermoelectric films. The results show that when the annealing temperature is higher than $800{ }^{\circ} \mathrm{C}$ crystallized $\mathrm{Ca}_{3} \mathrm{Co}_{4} \mathrm{O}_{9}$ films can be obtained on single-crystal $\mathrm{LaAlO}_{3}$ substrates, whereas the $\mathrm{Ca}_{3} \mathrm{Co}_{4} \mathrm{O}_{9}$ films are amorphous for lower annealing temperatures. Experimental evidence shows that the amorphous $\mathrm{Ca}_{3} \mathrm{Co}_{4} \mathrm{O}_{9}$ films behave like semiconductors with enhanced resistivity and Seebeck coefficient within the measured temperature range. The magnetoresistance is positive indicating the absence of long-range order of the Co-ion spins. For the crystallized $\mathrm{Ca}_{3} \mathrm{Co}_{4} \mathrm{O}_{9}$ films, it can be seen that the thinner films show the characteristics of spontaneous dewetting induced by the stress, which suggests that it is possible to prepare nanostructured $\mathrm{Ca}_{3} \mathrm{Co}_{4} \mathrm{O}_{9}$ films through tailoring the film thickness and the lattice mismatch; whereas, for the thicker films the temperature dependence of the resistivity and the Seebeck coefficient shows that it is possible to prepare excellent $\mathrm{Ca}_{3} \mathrm{Co}_{4} \mathrm{O}_{9}$ thermoelectric films using chemical solution deposition route, which will broaden the range of preparation methods for misfit structured Co-based thermoelectric films.
\end{abstract}

(C) 2007 American Institute of Physics. [DOI: 10.1063/1.2818109]

\section{INTRODUCTION}

Thermoelectric (TE) generation systems can convert heat energy directly into electrical energy, irrespective of the source size, which provides an effective route to use the waste heat from automobiles, factories, and so on. The TE generation systems require that the TE materials should possess high conversion efficiency and also be composed of nontoxic and abundantly available elemental materials having high chemical stability in air, even at temperatures of $800-1000 \mathrm{~K}$. As compared with the Bi-Te based TE materials, recently, the layered cobalt-based oxide TE materials have been widely studied because of their excellent TE properties, especially for applications involving higher temperatures and an oxidizing atmosphere. Among various cobaltbased oxide TE materials, $\mathrm{Ca}_{3} \mathrm{Co}_{4} \mathrm{O}_{9}(\mathrm{CCO} 349)$ has its dimensionless figure of TE merit $\mathrm{ZT}=S^{2} T / \rho \kappa$ (where $S$ is the Seebeck coefficient, $T$ is the temperature, $\rho$ is the electrical resistivity, and $\kappa$ is the thermal conductivity) about 1 near $1000 \mathrm{~K}$, which suggests that the CCO349 can be considered as a very important candidate for TE applications. ${ }^{1-3}$ The

\footnotetext{
${ }^{a)}$ Author to whom correspondence should be addressed. Electronic mail: xbzhu@issp.ac.cn or xuebin@uow.edu.au.
}

CCO349 is built up by stacking along the $c$-axis of triple rock salt-type layer $\mathrm{Ca}_{2} \mathrm{CoO}_{3}$ and single $\mathrm{CdI}_{2}$-type $\mathrm{CoO}_{2}$ layers, which both have monoclinic symmetry with identical $a$, $c$, and $\beta$, but different $b$ parameters (for the $\mathrm{Ca}_{2} \mathrm{CoO}_{3}$ layer, $a=4.8376 \AA, c=10.833 \AA, \beta=98.06^{\circ}$, and $b_{1}=4.5565 \AA$, while for the $\mathrm{CoO}_{2}$ layer, $\left.b_{2}=2.8189 \AA\right){ }^{4}$

As for the fabrication of $\mathrm{CCO} 349$ films, which can be used in various applications ${ }^{5}$ such as thermochemistry-on-achip, biothermoelectric chips, active cooling for microelectronic processors, and localized cooling/heating at points of interest, the commonly used method is pulsed laser deposition (PLD) ${ }^{6-8}$ and there have few reports about the chemical solution deposition (CSD) route, ${ }^{9}$ which is a simple, low-cost method for large-area films. Moreover, in the processes of preparing films, the interfacial strain in the films is a key parameter controlling many physical properties, which has stimulated considerable efforts towards understanding the relaxation mechanisms. ${ }^{10,11}$ Furthermore, the interfacial strain is the driving force for the generation of low-dimensional self-assembled nanostructures or for the spontaneous formation of discontinuous films through temperature-induced dewetting phenomena, which is considered as a promising 
methodology for the fabrication of new devices based on nanostructures such as nanodots or nanowires. ${ }^{12-16}$

In this article, we report on CSD of CCO349 films on $\mathrm{LaAlO}_{3}$ (LAO) single crystal substrates and the dewetting effects associated with strains are also studied. The results show that CCO349 films can be prepared using the simple CSD method on LAO substrates, although the mismatch is several tens of percentage points, which can broaden selection of substrates, as well as opening up a simple route for the preparation of CCO349 films, especially for large-scale production.

\section{EXPERIMENTAL}

CCO349 films were prepared by the CSD method. Caacetate (Alfa Aesar) and Co-acetate (Alfa Aesar) were dissolved in propionic acid at $70{ }^{\circ} \mathrm{C}$ and stirred at this temperature for $20 \mathrm{~min}$, and then the solution was stirred at room temperature for more than $10 \mathrm{~h}$ in order to get a well mixed precursor solution. The solution was diluted only by propionic acid to $0.7 \mathrm{M}$ in cations. At this stage the obtained solution can be kept for several months in atmosphere.

Before the process of deposition, the LAO substrates were ultrasonically cleaned with acetone for $15 \mathrm{~min}$ and then annealed at $900{ }^{\circ} \mathrm{C}$ for $60 \mathrm{~min}$ in air in order to obtain a well-crystallized LAO substrate surface. The films were prepared by the spin-coating method on LAO $(h 00)$ substrates using a rotation speed of $4500 \mathrm{rpm}$ and a time of $60 \mathrm{~s}$. The deposited films were then dried at $350{ }^{\circ} \mathrm{C}$ for $30 \mathrm{~min}$ in order to expel the organics. In order to obtain films with different thicknesses as well as study the dewetting phenomena, the spin-coating and drying procedures were repeated for different times from one to four times. Finally, the dried films with different thickness were annealed at different temperatures from 400 to $900{ }^{\circ} \mathrm{C}$ for $2 \mathrm{~h}$ under flowing oxygen with a flux of 450 SCCM (SCCM denotes cubic centimeter per minute at STP).

In order to study the kinetic process in the CSD for CCO349 films, films with two coatings were annealed at $900{ }^{\circ} \mathrm{C}$ for different times from 1 to $4 \mathrm{~h}$. Additionally, in order to give further images about the thickness effect on the dewetting phenomena, thinner $\mathrm{CCO} 349$ films were fabricated using more diluted solution with $0.3 M$ in cations and annealed at 800 and $900{ }^{\circ} \mathrm{C}$ for $2 \mathrm{~h}$.

In order to briefly study the mismatch effect on the dewetting, one $\mathrm{CCO} 349$ film with two coatings was fabricated on the $\gamma$-cut $\mathrm{Al}_{2} \mathrm{O}_{3}(0001)$ substrate and annealed at $850{ }^{\circ} \mathrm{C}$ for $2 \mathrm{~h}$. The lattice mismatch between the $\mathrm{Al}_{2} \mathrm{O}_{3}$ and $\mathrm{CCO} 349$ is $1.5 \%$ for the $\mathrm{CoO}_{2}$ layer $/ \mathrm{Al}_{2} \mathrm{O}_{3}$ and $4.5 \%$ for the $\mathrm{Ca}_{2} \mathrm{CoO}_{3}$ layer $/ \mathrm{Al}_{2} \mathrm{O}_{3}{ }^{6}$

The CCO349 film was also prepared on Si (100) substrate with native $\mathrm{SiO}_{2}$ layer using the solution with concentration of $0.7 \mathrm{M}$ in cations and $850{ }^{\circ} \mathrm{C}$ annealing temperature for $2 \mathrm{~h}$ in order to study the substrate-induced stress effect on the metal-insulator transition.

Thermogravimetric (TG) measurements on the CCO349 powder obtained from the solution dried at $100{ }^{\circ} \mathrm{C}$ were carried out on a Perkin-Elmer designed Pyris Diamond differential scanning calorimeter (DSC). X-ray diffraction (XRD)

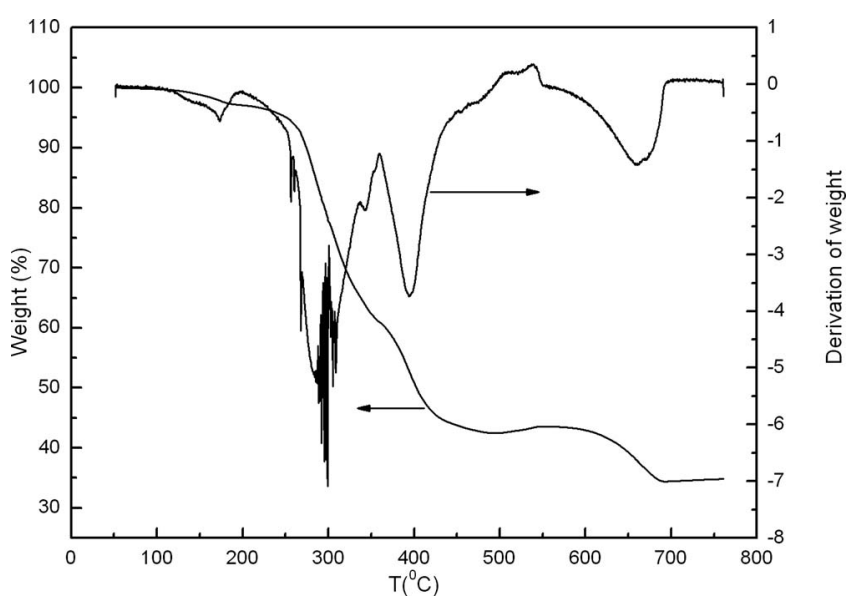

FIG. 1. TG and DTA results on CCO349 powders in air.

was carried out at different temperatures using a Philips X'pert Pro diffractometer with $\mathrm{Cu} K \alpha$ radiation to check the crystallization and orientation both for out of plane and inplane. Field-emission scanning electronic microscopy (FESEM) (FEI Sirion 200) and atomic force microscopy (AFM) (Park Scientific Instruments Autoprobe CP) were carried out to check the microstructure and the thickness. The thickness was checked by the cross section of the FE-SEM, and it was observed that the one layer CCO349 film was consisted of discontinuous CCO349 islands with the height from 50 to $100 \mathrm{~nm}$; while, the island height of CCO349 film was about 100-200 nm for the two layer and three layer CCO349 films, however, the island distance was decreased with the increasing of the layers; and the thickness of the four layer CCO349 film was about 200-220 nm. The resistivity data were obtained using a physical properties measurement system (PPMS) (Quantum Design) with a standard four-point probe method, and the Seebeck coefficient was measured using PPMS in the four-probe steady state mode.

\section{RESULTS AND DISCUSSIONS}

Figure 1 shows the TG and the derivative of the TG (DTA) results within the measured temperature range of $50-750{ }^{\circ} \mathrm{C}$. It can be seen that at $300{ }^{\circ} \mathrm{C}$ the weight is greatly decreased, which can be attributed to the volatilization of the organic solvents. When the temperature is increased to $700{ }^{\circ} \mathrm{C}$ the weight loss is slight. From Fig. 2, it can be seen that the film annealed at $750{ }^{\circ} \mathrm{C}$ in oxygen is amorphous without any CCO349 diffraction peaks. Moreover, no intermediate phases are detected by synchronous XRD measurements from room temperature to $750{ }^{\circ} \mathrm{C}$. When the annealing temperature is increased to $800{ }^{\circ} \mathrm{C}$, one can clearly see that the derived films are highly $c$-axis oriented without any other undesired diffraction peaks. However, when the annealing temperature is increased to $925^{\circ} \mathrm{C}$, some undesired peaks appear, which suggests that in our experiments the stable annealing temperature range for crystallized $\mathrm{CCO} 349$ films is $800-900{ }^{\circ} \mathrm{C}$. Additionally, the $c$-axis lattice constant is about $10.70 \AA$ for all the crystallized films, which is smaller than that of the bulk material lattice constant ( 10.83 $\AA$ ) (Ref. 4) and approaches the lattice constant of the CCO349 films prepared by PLD (10.70 $\AA$ ), indicating 


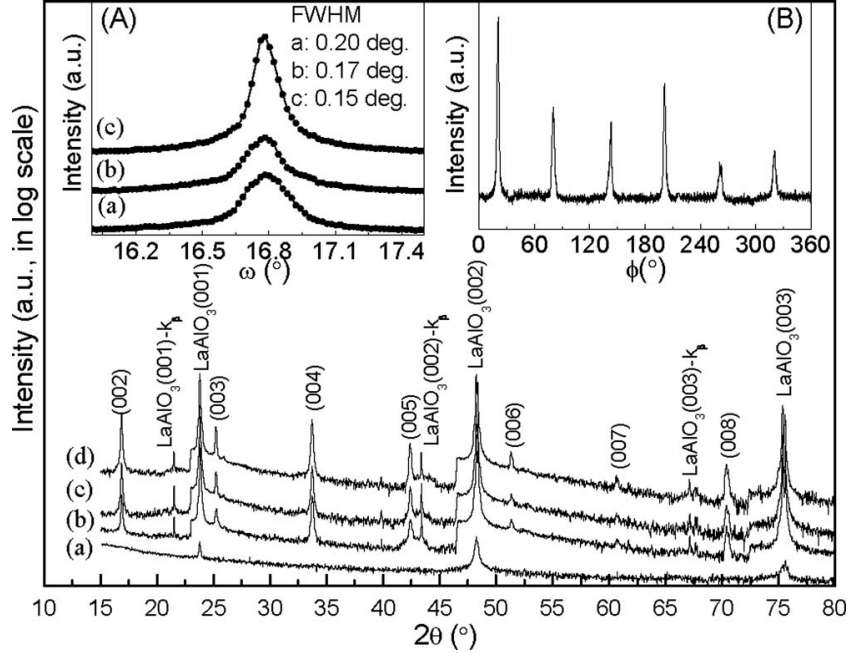

FIG. 2. XRD of $\mathrm{CCO} 349 / \mathrm{LAO}$ annealed at different temperatures: (a) $700{ }^{\circ} \mathrm{C}$, (b) $800{ }^{\circ} \mathrm{C}$, (c) $850{ }^{\circ} \mathrm{C}$, and (d) $900{ }^{\circ} \mathrm{C}$. The inset (A) shows the (004)-plane rocking curves for films annealed at different temperatures: (a) $800{ }^{\circ} \mathrm{C}$, (b) $850{ }^{\circ} \mathrm{C}$, and (c) $900{ }^{\circ} \mathrm{C}$. The inset (B) is the typical $\Phi$-scanning result on the $900{ }^{\circ} \mathrm{C}$-annealed $\mathrm{CCO} 349$ film with two coatings.

that the derived $\mathrm{CCO} 349$ films are in-plane tensile strained. ${ }^{5,7}$ A similar shortening of the $c$-axis lattice constant for all the films suggests that there have been the same inplane tensile stress for all derived films, which can be attributed to the dewetting and will be discussed below.

The lattice mismatch $\Delta a=\left(a_{f}-a_{s}\right) / a_{f} \times 100 \%$, where $a_{f}$ and $a_{s}$ are the $\mathrm{CoO}_{2}$-layer and the substrate lattice constants, respectively, and is $+21 \%$ and $-36 \%$ for the $a$-axis and $b$-axis, respectively. It is interesting to observe that highly $c$-axis oriented CCO349 films can be obtained on even such largely lattice-mismatched substrates, which will broaden the choice of substrates and can be explained according to the near coincidence site lattice (NCSL) theories. ${ }^{17}$

In order to check the out-of-plane orientation of the prepared CCO349 films, rocking curve measurements were carried out for the films with one coating but annealed at different temperatures, and the results are shown in the inset (A) of Fig. 2. It can be clearly seen that with increasing annealing temperature the full width at half maximum (FWHM) of the rocking curve is decreased from $0.20^{\circ}$ to $0.15^{\circ}$, meaning an improvement in the out-of-plane orientation, which can be attributed to an increase in the driving energy for epitaxial/ oriented growth. ${ }^{15}$

Figure 3 presents the FE-SEM results on films with two coatings that were annealed at different temperatures. It can be observed that when the annealing temperature is lower than $800{ }^{\circ} \mathrm{C}$ the films are dense and show amorphous characteristics, which is the same as the XRD results. When the annealing temperature is increased to $800{ }^{\circ} \mathrm{C}$, it is observed that the films are dewetting. Moreover, as the annealing temperature increases, the dewetting area appeared as white color in the FE-SEM results and is also increased. The energy dispersive spectroscopy (EDS) analysis unambiguously indicates that the dewetting area lacks the $\mathrm{Ca}$ and Co elements, and only the $\mathrm{La}$ and $\mathrm{Al}$ elements of the $\mathrm{LAO}$ substrate are present. Moreover, the ratio of $\mathrm{Ca} / \mathrm{Co}$ in the wetting area is about 1.3 , implying that the derived films are stoichio-
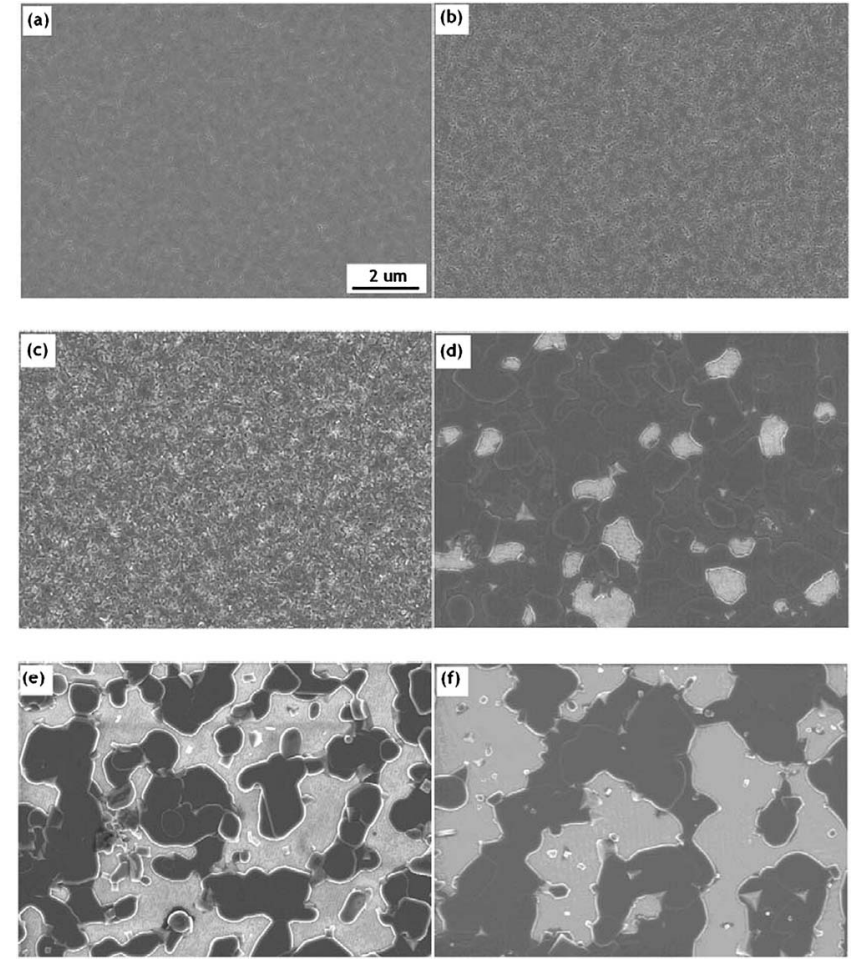

FIG. 3. FE-SEM results on $\mathrm{CCO} 349$ films annealed at different temperatures: (a) $500{ }^{\circ} \mathrm{C}$, (b) $600{ }^{\circ} \mathrm{C}$, (c) $700{ }^{\circ} \mathrm{C}$, (d) $800{ }^{\circ} \mathrm{C}$, (e) $850{ }^{\circ} \mathrm{C}$, and (f) $900{ }^{\circ} \mathrm{C}$

metric. Figures 4(a)-4(c) present the three-dimensional (3D) AFM results on several typical films that show dewetting. It can be seen that as the annealing temperature increases, the dewetting is obviously enhanced, which is same as the results from FE-SEM. Moreover, from Fig. 4(d) one can see that the wetting area is about $100-200 \mathrm{~nm}$ in thickness and that the lateral face is nearly normal to the substrate and can be attributed to the $\{100\}$ planes of CCO349. It is believed that the dewetting phenomena should be appeared in the epitaxial films or biaxially textured film. In order to check the in-plane orientation of the derived films, XRD phi-scanning measurements ${ }^{6}$ were carried out for the $\mathrm{CCO} 349$ films with two coatings but annealed at different temperatures. It is observed that all films are biaxially textured and six diffraction
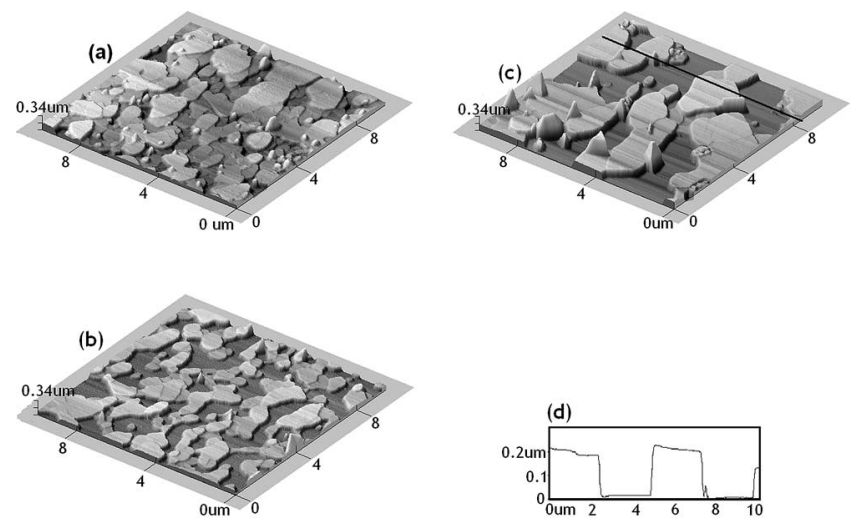

FIG. 4. 3D-AFM results on CCO349 films with two coatings annealed at different temperatures: (a) $800{ }^{\circ} \mathrm{C}$, (b) $850{ }^{\circ} \mathrm{C}$, (c) $900{ }^{\circ} \mathrm{C}$, and (e) profile image for $900^{\circ} \mathrm{C}$-annealed film along the line in the (c). 


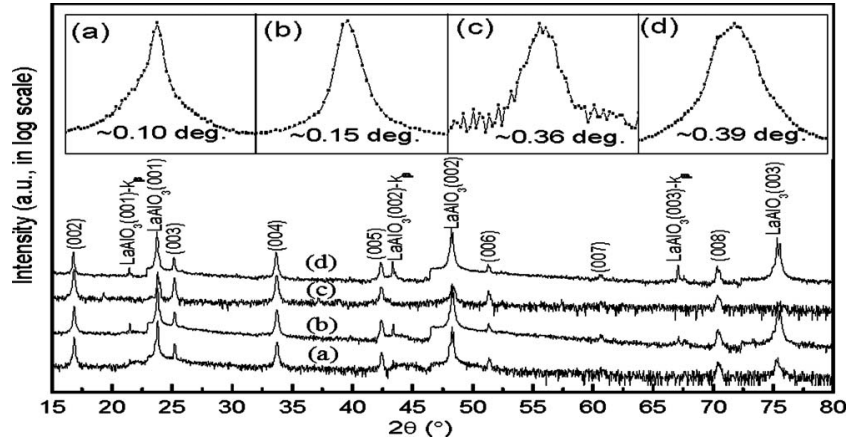

FIG. 5. XRD $\theta-2 \theta$ results on CCO349 films annealed at $900{ }^{\circ} \mathrm{C}$ but different coatings: (a) one coating, (b) two coatings, (c) three coatings, and (d) four coatings. The inset shows the (004)-plane rocking curves of CCO349 films annealed at $900{ }^{\circ} \mathrm{C}$ but composed of different coatings: (a) one coating, (b) two coatings, (c) three coatings, and (d) four coatings.

peaks are clearly observed. The typical phi-scanning result for the $900{ }^{\circ} \mathrm{C}$-annealed CCO349 film with two coatings are shown in the inset (B) of Fig. 2, which clearly suggests that the derived films have in-plane orientation.

In order to study the thickness effects on the dewetting, we carried out the preparation of CCO349 films with different coatings. The XRD results on the $900{ }^{\circ} \mathrm{C}$-annealed films with different coatings are shown in Fig. 5. It can be seen that all the obtained films are also highly $c$-axis oriented without any undesired diffraction peaks. The $c$-axis lattice constants are also $10.70 \AA$ for all the films despite the thickness variations, meaning that the interface stress is also the same for all the films. The out-of-plane orientation of the $\mathrm{CCO} 349$ films annealed at $900{ }^{\circ} \mathrm{C}$ with different coatings was also investigated through rocking curve measurements, and the results are also shown in Fig. 5. The results clearly suggest that the out-of-plane orientation of the film is decreased as the film thickness increases, which can be attributed to the improved bulk nucleation within the bulk films. ${ }^{15}$

Figures 6(a)-6(d) presents the FE-SEM results on the films annealed at $850^{\circ} \mathrm{C}$ with different coatings. It is found that with increasing thickness the dewetting area is decreased, and the films with four coatings are dense without dewetting. Moreover, for the films with four coatings, many hexagonal grains stay on the bottom layer with sharply flat morphology. The mean size of the hexagonal grains in the $900{ }^{\circ} \mathrm{C}$-annealed film as shown in Fig. 6(f) is larger than that of the $850{ }^{\circ} \mathrm{C}$-annealed film as shown in Fig. 6(e), which can be attributed to the enhanced atomic diffusion.

In order to further study the influence of the thickness on the dewetting effect, thinner CCO349 films derived from a diluted solution ( $0.3 \mathrm{M}$ in cations) were prepared using 800 and $900{ }^{\circ} \mathrm{C}$ annealing temperatures. The FE-SEM results are shown in Figs. 7(a) and 7(b). It can be clearly seen that the dewetting area is increased as compared with the thicker films. Moreover, it is observed that the grain size of the film annealed at $900{ }^{\circ} \mathrm{C}$ is larger than that of the film annealed at $800{ }^{\circ} \mathrm{C}$, proving that the grain size increases with increasing annealing temperatures. From the above results, it is expected that if the solution concentration is further decreased, one can obtain highly oriented CCO349 films with nano-
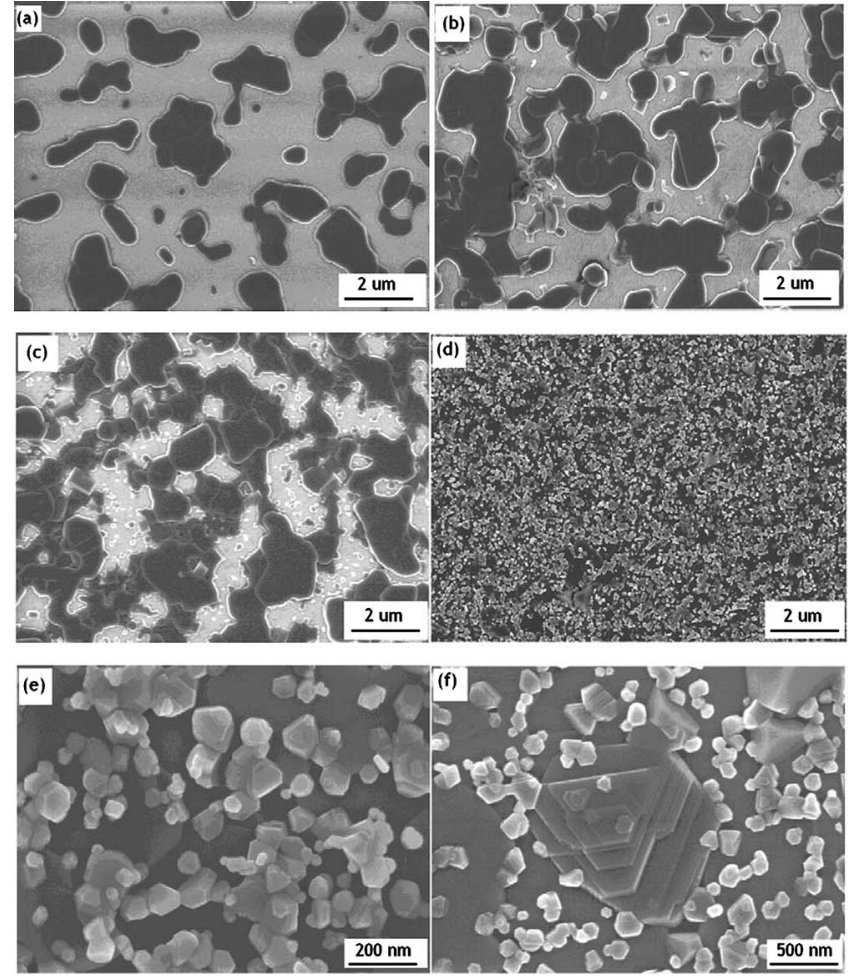

FIG. 6. FE-SEM results on CCO349 films annealed at $850{ }^{\circ} \mathrm{C}$ but with different coatings: (a) one coating, (b) two coatings, (c) three coatings, and (d) four coatings. (e) and (f) are FE-SEM with high magnification for four coatings annealed at 800 and $900{ }^{\circ} \mathrm{C}$, respectively.
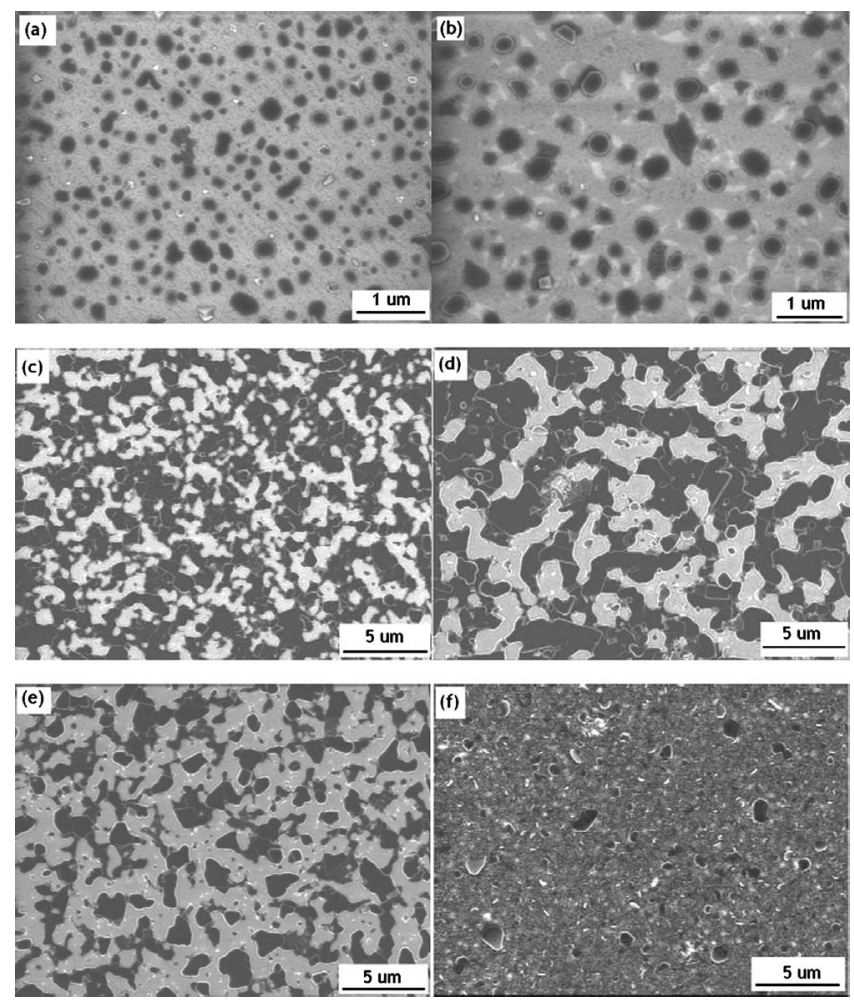

FIG. 7. (a) and (b) are the FE-SEM results on films derived from a $0.3 M$ solution and annealed at 800 and $900{ }^{\circ} \mathrm{C}$, respectively. (c)-(e) are the FESEM results on films with two coatings that were annealed at $900{ }^{\circ} \mathrm{C}$ but for different times: (c) $1 \mathrm{~h}$, (d) $2 \mathrm{~h}$, and (e) $4 \mathrm{~h}$. (f) is the FE-SEM result for a CCO349 film annealed at $850{ }^{\circ} \mathrm{C}$ with two coatings on $\mathrm{Al}_{2} \mathrm{O}_{3}$. 
structured particles on LAO substrates, which will open up a simple route to CCO349 films with nanostructures.

We should now consider if the spontaneously generated surface structures described above are in a quasiequilibrium state or, instead, if they are kinetically limited structures. This is a key issue because it is intimately related to the basis for modeling the observed behavior. In addition, it will open up the possibility to generate different nanostructures controlled by growth kinetics and coarsening effects. Based on the above consideration, we have investigated the influence of the annealing times on the surface structure of the CCO349 films. The FE-SEM results on the $900{ }^{\circ} \mathrm{C}$-annealed films with two coatings but different annealing times are shown in Figs. 7(c)-7(e). It can be clearly seen that the dewetting area increases with the annealing time. It can be then concluded that the structure of the CCO349 film with two coatings is a metastable state that evolves toward a new equilibrium state through atomic diffusion process. That is to say, during the growth process of the CCO349 films, the nucleation and growth of pinholes or cracks, which are the origin of the dewetting, are propagated. Moreover, there is no dewetting for thicker films with four coatings, even if the annealing period is prolonged to longer than $4 \mathrm{~h}$.

In order to investigate the lattice mismatch effects on the dewetting, $\mathrm{CCO} 349$ films on $\gamma$-cut $\mathrm{Al}_{2} \mathrm{O}_{3}$ (0001) substrates were prepared and the lattice mismatch between the $\mathrm{Al}_{2} \mathrm{O}_{3}$ and $\mathrm{CCO} 349$ is $1.5 \%$ for the $\mathrm{CoO}_{2}$ layer $/ \mathrm{Al}_{2} \mathrm{O}_{3}$ and $4.5 \%$ for the $\mathrm{Ca}_{2} \mathrm{CoO}_{3}$ layer $/ \mathrm{Al}_{2} \mathrm{O}_{3}$, which is obviously smaller than that of the CCO349 films on LAO substrates. Figure 7(f) is the typical FE-SEM result for the $\mathrm{CCO} 349$ film $/ \mathrm{Al}_{2} \mathrm{O}_{3}$ with two coatings and annealed at $850{ }^{\circ} \mathrm{C}$ for $2 \mathrm{~h}$; it is seen that the film shows no characteristics of dewetting, which suggests that the lattice mismatch plays an important role on the determination of the dewetting phenomena.

It is often observed that the dewetting occurs only when the thickness of a film is lower than a critical value. ${ }^{18}$ For our experiments, if we define the island geometry as parallepipedic and the crystal structure of the CCO349 is monoclinic, we can define the relevant interfacial and surface energies as follows: $\gamma_{001}, \gamma_{100}, \gamma_{i}$, and $\gamma_{s}$ are the corresponding energies for the surface energy of the CCO349 (001) plane, the surface energy of the $\mathrm{CCO} 349\{100\}$ plane, the interfacial energy of the film/substrate, and the surface energy of the substrate, respectively. Then, performed the same as in Ref. 14, we can write the total energy $E_{0}$ for a homogeneous continuous film (without dewetting such as the four layer CCO349 films) with a surface $A=a^{2}$ and the total energy $E$ for a dewetted film. The $E / E_{0}$ as a function of the normalized dimension $x$ of the dewetted areas in the film having an initial thickness $h_{0}$ can be normalized to $\gamma_{001}$, and the obtained equation is as follows:

$$
\begin{aligned}
E= & \frac{E}{E_{0}}=\left(1-x^{2}\right)+\frac{x h_{0}}{a\left(1-x^{2}\right)}\left(\frac{\left(\gamma_{100} / \gamma_{001}\right)}{1+\gamma_{i} / \gamma_{001}}\right) \\
& +x^{2}\left(\frac{\left(\gamma_{s} / \gamma_{001}\right)}{\gamma_{i} / \gamma_{001}}\right)
\end{aligned}
$$

Since we have no detailed datum about the $\gamma_{s} / \gamma_{001}$ and $\gamma_{100} / \gamma_{001}$, considering the layered structure of the CCO 349 ,
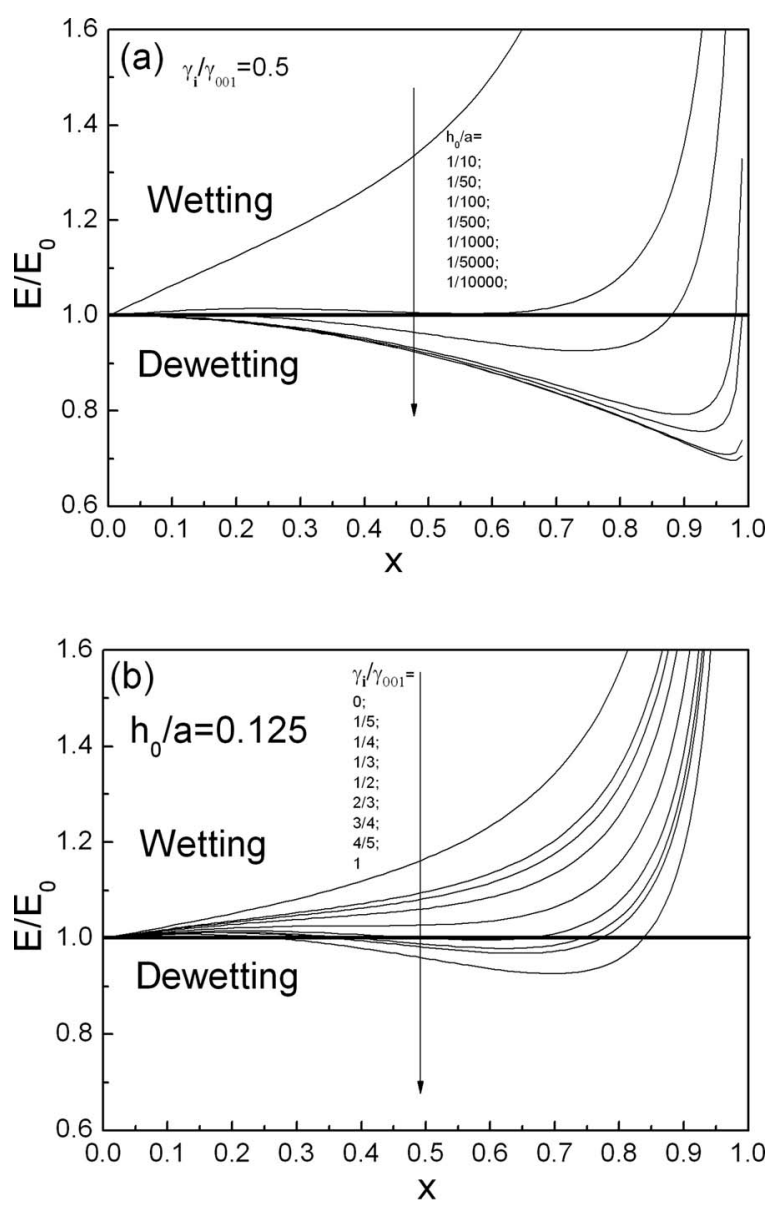

FIG. 8. (a) is the typical case for $E / E_{0}$ with fixed $\gamma_{i} / \gamma_{001}=0.5$ but different initial thicknesses $h_{0} / a$; (b) shows the typical case for $E / E_{0}$ with fixed $h_{0} / a=0.125$ but different $\gamma_{i} / \gamma_{001}$.

we assume that $\gamma_{s} / \gamma_{001} \approx 1$ and $\gamma_{100} / \gamma_{001} \approx 10$, then we can estimate the relative stability of the different surface configurations. Figure 8(a) is the typical case for $E / E_{0}$ with fixed $\gamma_{i} / \gamma_{001}=0.5$ but different initial thicknesses $h_{0} / a$, and Fig. 8(b) shows the typical case for $E / E_{0}$ with fixed $h_{0} / a$ $=0.125$ but different $\gamma_{i} / \gamma_{001}$. From Fig. 8(a) it can be seen that when the thickness is below a certain value, the CCO349 film becomes unstable, i.e., $E / E_{0}<1$; moreover, the $x_{\min }$ increases for thinner films, meaning that the $\mathrm{CCO} 349$ film becomes less stable when the thickness is decreased, which corresponds to the CCO349 films on LAO substrates with different coatings. From Fig. 8(b) it can be seen that when $\gamma_{i} / \gamma_{001}$ is higher than a certain value, the CCO349 film becomes unstable; moreover, with the increase of $\gamma_{i} / \gamma_{001}$ the $x_{\min }$ increases, which suggests that the dewetting area is improved when the interfacial energy is increased, corresponding to the conditions of CCO349 films on $\mathrm{LAO}$ and $\mathrm{Al}_{2} \mathrm{O}_{3}$ substrates. The above results clearly suggest that it is possible to prepare nanostructured $\mathrm{CCO} 349$ films through tailoring the film thickness and the lattice mismatch.

As a final experimental investigation intending to quantify the prepared CCO349 films, we have carried out measurements of the temperature dependence of the resistivity $(\rho-T)$ and Seebeck coefficient $(S-T)$. Figure 9 presents the typical $\rho-T$ results for the films without dewetting. It can be seen that the amorphous film behaves as a semiconductor 

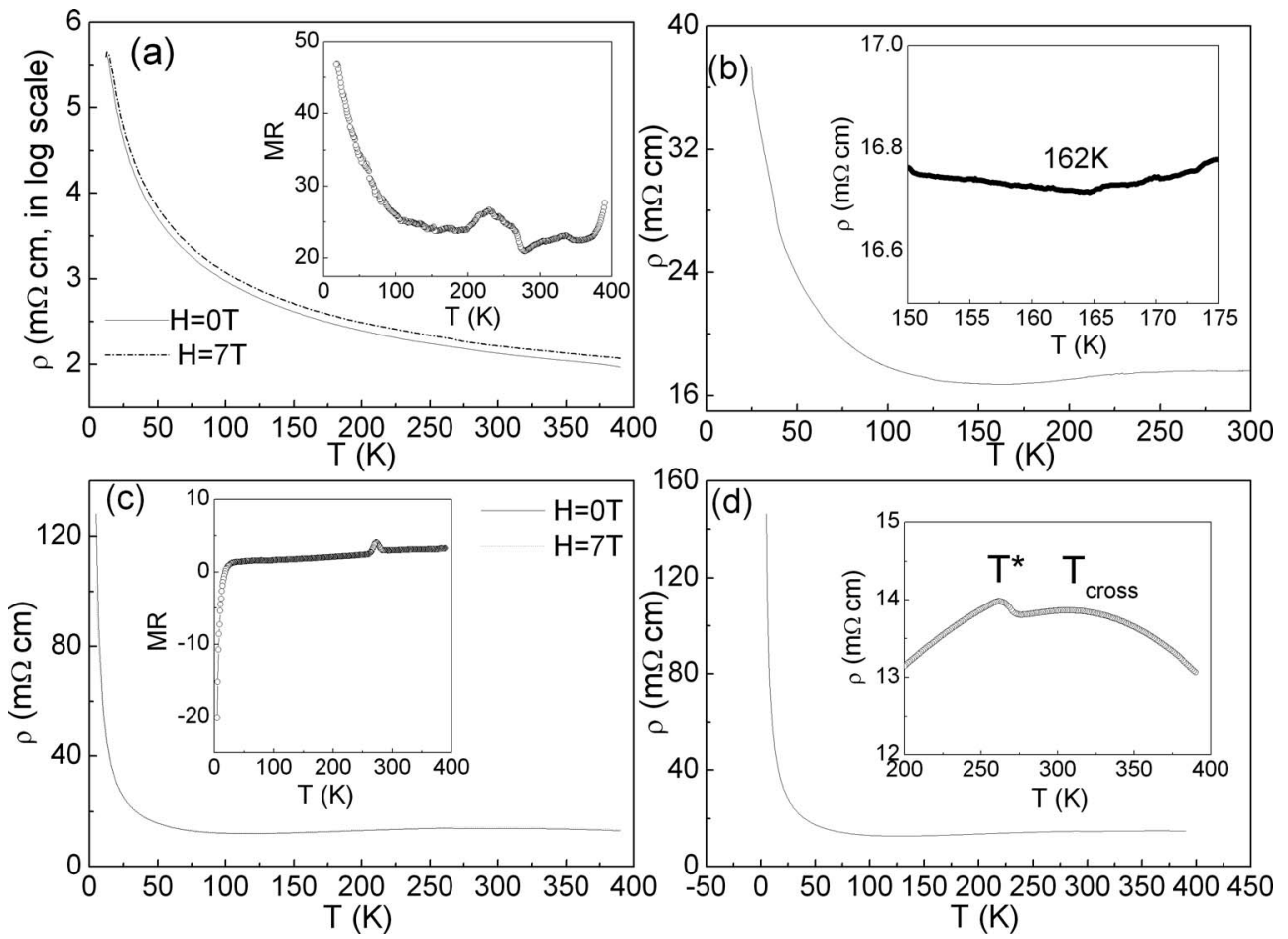

FIG. 9. $\rho$ - $T$ results on different films. (a) $700{ }^{\circ} \mathrm{C}$-annealed film with two coatings; the inset contains the corresponding magnetoresistance results. (b) $800{ }^{\circ} \mathrm{C}$-annealed film with four coatings; the inset shows the enlarged result. (c) $850^{\circ} \mathrm{C}$-annealed film with four coatings; the inset contains the corresponding magnetoresistance. (d) $900{ }^{\circ} \mathrm{C}$-annealed film with four coatings; the inset shows the enlarged results at higher temperatures.

with enhanced resistivity; whereas, all the crystallized films have an insulator-metal (IM) transition near 100-200 K, as shown in the inset of Fig. 9(b), which is similar to the results on single crystals, ${ }^{19}$ bulk ceramics, ${ }^{4}$ and films prepared by PLD (Refs. 6-8) and can be attributed to the spin-densitywave (SDW) effects. However, the IM transition temperature is higher than that of the bulk CCO349 samples, ${ }^{4}$ which may be related to the residual stress in the derived films. ${ }^{20}$ In order to verify this presumption we have prepared $\mathrm{CCO} 349$ films on $\mathrm{Si}$ substrates with a native $\mathrm{SiO}_{2}$ amorphous layer. The XRD results on the $\mathrm{CCO} 349 / \mathrm{SiO}_{2} / \mathrm{Si}$ film annealed at $850{ }^{\circ} \mathrm{C}$ with four coatings is shown in inset (a) of Fig. 10, which gives the evidence that the film is randomly oriented,

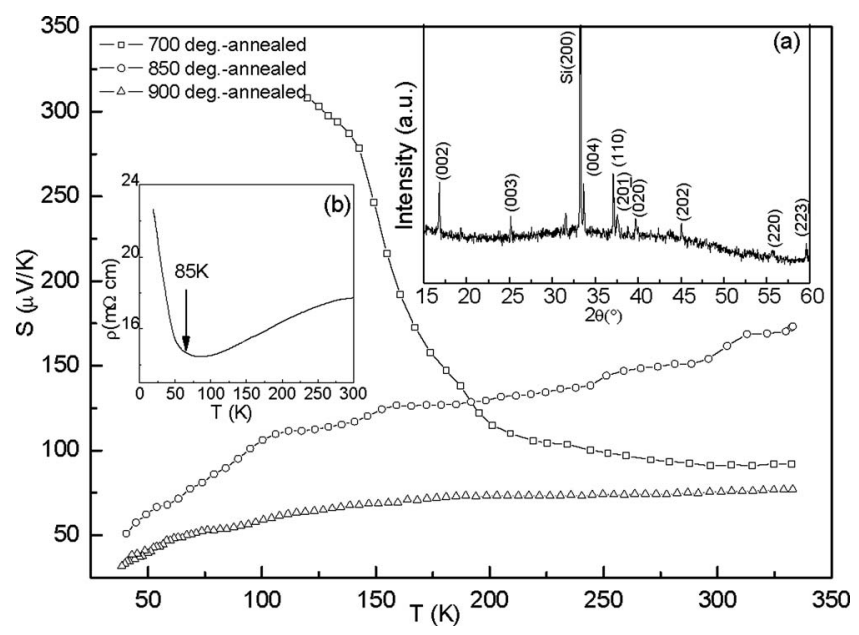

FIG. 10. $S$ - $T$ results on different films. Inset (a) contains the XRD results on $\mathrm{CCO} 349 / \mathrm{SiO}_{2} / \mathrm{Si}$, and inset (b) is the $\rho$ - $T$ results on $\mathrm{CCO} 349 / \mathrm{SiO}_{2} / \mathrm{Si}$. meaning that there is no residual stress in this sample. The $\rho$ - $T$ result for the $\mathrm{CCO} 349 / \mathrm{SiO}_{2} / \mathrm{Si}$ film is shown in inset (b) of Fig. 10, which shows that the IM transition temperature is about $85 \mathrm{~K}$, similar to the results on CCO349 ceramics $^{4}$ or single crystals, ${ }^{19}$ meaning that the enhanced IM temperature of the CCO349 films on LAO substrate can be attributed to the residual stress effects. Moreover, for all the crystallized films it is observed that the resistivity at $300 \mathrm{~K}$ is about $10-20 \mathrm{~m} \Omega \mathrm{cm}$, which is slightly larger than that of the films derived by PLD, but is similar to that of the films prepared by CSD, ${ }^{9}$ which can be attributed to large amounts of defects in the CSD-derived films. For the CCO349/LAO films with different layers, it should be pointed out that the resistivity is very large and cannot be measured in the PPMS for the one layer film, and the resistivity at $300 \mathrm{~K}$ of the two and three layer films is $\sim 50-100$ and $30-50 \mathrm{~m} \Omega \mathrm{cm}$, respectively, which means that the percolation effect also plays an important role on the resistivity. Moreover, from the enlarged $\rho-T$ curve as shown in the inset of Fig. 9(d), one can also observe that there is a transition located at $T_{\text {cross }}$ that is attributed to the Co-ion spin state crossover. ${ }^{4}$ Additionally, above $T_{\text {cross }}$, there is another IM transition denoted as $T^{*}$, which corresponds roughly to the Mott limit $k_{F} l=\left(h / e^{2}\right) c / \rho$, with the Fermi wave vector $k_{F}$ and the mean free path $l(c=10.70 \AA) .{ }^{19}$

In order to check the effects of the applied magnetic field on the properties of the prepared films, the $\rho-T$ under $7 \mathrm{~T}$ was measured. The temperature dependence of the magnetoresistance (MR) $\left[\left(\rho_{7 T}-\rho_{0 T}\right) / \rho_{0 T} \times 100 \%\right]$ for typical films is plotted in the inset of Fig. 9(a) and the inset of Fig. 9(c). It can be seen that for the film annealed at $850{ }^{\circ} \mathrm{C}$ the MR is 
negative and positive for $T<20 \mathrm{~K}$ and $T>20 \mathrm{~K}$, respectively, which is related to the ferrimagnetism and the Lorenz force, respectively. ${ }^{4}$ For the amorphous film, the results show that the MR is positive within the whole measured temperature range, which suggests that the ferrimagnetic order cannot be formed in amorphous film.

For TE materials, one important parameter is the Seebeck coefficient $S$. In our experiments, $S$ is measured using the four-probe steady state mode on PPMS, and the results are shown in Fig. 10. It can be seen that for the crystallized films, the $S(T)$ behaves as metals, whereas, for the amorphous film the $S(T)$ is semiconductorlike. Moreover, the $S(300 \mathrm{~K})$ is $\sim 154$ and $\sim 80 \mu \mathrm{V} / \mathrm{K}$ for the films annealed at 850 and $900{ }^{\circ} \mathrm{C}$ on LAO, respectively; the $S$ value at low temperatures is improved larger than $300 \mu \mathrm{V} / \mathrm{K}$ for the amorphous film, which can be attributed to the enhanced effective mass. ${ }^{9}$ In cobalt-based TE materials, it is usual to find that the $S$ is intimately relative to the Co-ion spin states. In $\mathrm{NaCo}_{2} \mathrm{O}_{4}$, if the low-spin (LS) states of $\mathrm{Co}^{3+}$ and $\mathrm{Co}^{4+}$ are stable, the $g_{3}$ and $g_{4}$ will be 1 and 6 , where $g_{3}$ and $g_{4}$ are the numbers of the configurations of the $\mathrm{Co}^{3+}$ and $\mathrm{Co}^{4+}$ ions, respectively; when the LS states of a $\mathrm{Co}^{3+}$ are stable and the LS and high-spin (HS) states of $\mathrm{Co}^{4+}$ are close in energy, the $g_{3}$ and $g_{4}$ will be 1 and $12 .^{21}$ Additionally, a photoemission study has revealed that the ratio of $\mathrm{Co}^{3+} / \mathrm{Co}^{4+}$ is $1: 2 .{ }^{22}$ If the above two spin states are possible, then the $S$ can be estimated using the Heikes formula, and the results are 94 and $154 \mu \mathrm{V} / \mathrm{K}$, which is close to our results on CCO349/LAO film annealed at 900 and $850{ }^{\circ} \mathrm{C}$, respectively, suggesting that it is possible to tune the $S$ values through control of the annealing temperature in the CSD processing.

\section{CONCLUSION}

A simple chemical solution deposition using cheap precursors was used to prepare $\mathrm{Ca}_{3} \mathrm{Co}_{4} \mathrm{O}_{9}$ films on $\mathrm{LaAlO}_{3}$ single-crystal substrates. The results showed that when the thickness was lower than a certain value the dewetting phenomenon occurred, which originates from the large interface stress between the film and the substrate. The simulation results showed that it was possible to fabricate nanostructured $\mathrm{Ca}_{3} \mathrm{Co}_{4} \mathrm{O}_{9}$ films through tailoring the film thickness and the lattice mismatch. The microstructures and physical properties of the wetting films clearly suggest that chemical solution deposition can be used to prepare $\mathrm{Ca}_{3} \mathrm{Co}_{4} \mathrm{O}_{9}$ films, which opens up a simple route to fabricate large-area Cobased oxide thermoelectric films.

\section{ACKNOWLEDGMENTS}

This work is supported by a National Key Basic Research grant under Contract No. 2006CB601005, funding from the Chinese Academy of Sciences under Contract No. O64N161294, and funding from Australian Research Council under Contract No. DP 0666771.

${ }^{1}$ K. Koumoto, I. Terasaki, and R. Funahashi, MRS Bull. 31, 206 (2006).

${ }^{2}$ T. M. Tritt and M. A. Subramanian, MRS Bull. 31, 188 (2006).

${ }^{3}$ R. Funahashi, I. Matsubara, H. Ikuta, T. Takeuchi, U. Mazutani, and S. Sodeoka, Jpn. J. Appl. Phys., Part 2 39, L1127 (2000).

${ }^{4}$ A. C. Masset, C. Michel, A. Maignan, M. Hervieu, O. Toulemonde, F. Studer, and B. Raveau, Phys. Rev. B 62, 166 (2000).

${ }^{5}$ Y. F. Hu, W. D. Si, E. Sutter, and Q. Li, Appl. Phys. Lett. 86, 082103 (2005).

${ }^{6}$ H. W. Eng, W. Prellier, S. Herbert, D. Grebille, L. Michin, and B. Mercey, J. Appl. Phys. 97, 013706 (2005).

${ }^{7}$ Y. F. Hu, W. D. Si, E. Sutter, and Q. Li, Appl. Phys. Lett. 87, 171912 (2005).

${ }^{8}$ A. Pautrat, H. W. Eng, and W. Prellier, Phys. Rev. B 72, 233405 (2005).

${ }^{9}$ I. Matsubara, R. Funahashi, M. Shikano, K. Sasaki, and H. Enomoto, Appl. Phys. Lett. 80, 4729 (2002).

${ }^{10}$ K. Brunner, Rep. Prog. Phys. 65, 27 (2002).

${ }^{11}$ J. P. Locquet, J. Perret, J. Fompeyrine, E. Machler, J. W. Seo, and G. Van Tendeloo, Nature (London) 394, 453 (1998).

${ }^{12}$ V. A. Schukin and D. Bimberg, Rev. Mod. Phys. 71, 1125 (1999).

${ }^{13}$ C. Teichert, Phys. Rep. 365, 335 (2002).

${ }^{14}$ M. Coll, K. Gazquez, A. Pomar, K. Sandiumenge, and X. Obradors, Phys. Rev. B 73, 075420 (2006)

${ }^{15}$ R. W. Schwartz, T. Schneller, and R. Waser, C. R. Chim. 7, 433 (2004).

${ }^{16}$ I. Szafraniak, C. Harnagea, R. Scholz, S. Bhattacharyya, D. Hesse, and M. Alexe, Appl. Phys. Lett. 83, 2211 (2003).

${ }^{17}$ P. A. Langjahr, T. Wagner, F. F. Lang, and M. Ruhle, J. Cryst. Growth 256, 162 (2003).

${ }^{18}$ J. H. Kim and F. F. Lange, J. Mater. Res. 14, 1626 (1999).

${ }^{19}$ B. C. Zhao, Y. P. Sun, W. J. Lu, X. B. Zhu, and W. H. Song, Phys. Rev. B 74, 144417 (2005).

${ }^{20}$ P. Limelette, V. Hardy, P. Auban-Senzier, D. Jerome, D. Flahaut, S. Hebert, R. Fresard, Ch. Simon, J. Nouderm, and A. Maignan, Phys. Rev. B 71, 233108 (2005).

${ }^{21}$ W. Koshibae, K. Tsutsui, and S. Maekawa, Phys. Rev. B 62, 6869 (2000).

${ }^{22}$ S. Hirata, M.S. Thesis, University of Tokyo, 2004. 